\title{
HSCT may lower leukemia risk in ELANE neutropenia: a before-after study from the French Severe Congenital Neutropenia Registry
}

\author{
Gioacchino Andrea Rotulo ${ }^{1,2}$ - Blandine Beaupain ${ }^{1} \cdot$ Fanny Rialland $^{3}$ - Catherine Paillard ${ }^{4}$. Ouahiba Nachit ${ }^{1}$. \\ Claire Galambrun ${ }^{5}$. Virginie Gandemer ${ }^{6} \cdot$ Yves Bertrand $^{7} \cdot$ Benedicte Neven $^{8}$. Eric Dore ${ }^{9} \cdot$ Despina Moshous $^{10}$. \\ Bruno Filhon ${ }^{11}$. Nathalie Aladjdi ${ }^{12}$. Flore Sicre de Fontbrune ${ }^{13} \cdot$ Regis Peffault de la Tour $^{13} \cdot$ Marie Ouachee $^{7}$. \\ Christine Bellanne-Chantelot ${ }^{14} \cdot$ Jean-Hugues Dalle $^{15} \cdot$ Jean Donadieu $^{1}{ }^{1}$
}

Received: 25 July 2019 / Revised: 28 November 2019 / Accepted: 16 January 2020 / Published online: 28 January 2020

(c) The Author(s), under exclusive licence to Springer Nature Limited 2020

\begin{abstract}
ELANE neutropenia is associated with myelodysplasia and acute leukemia (MDS-AL), and severe infections. Because the MDS-AL risk has also been shown to be associated with exposure to GCSF, since 2005, in France, patients receiving high daily GCSF doses (>15 $\mu \mathrm{g} / \mathrm{kg} /$ day) are eligible for HSCT, in addition to classic indications (MDS-AL or GCSF refractoriness). We analyzed the effect of this policy. Among 144 prospectively followed ELANE-neutropenia patients enrolled in the French Severe Congenital Neutropenia Registry, we defined two groups according to period: "before 2005" for those born before 2005 and followed until 31/12/2004 (1588 person-years); and "after 2005" comprised of those born after 2005 or born before 2005 but followed after 2005 until 31/03/2019 (1327 person-years). Sixteen of our cohort patients underwent HSCT (14 long-term survivors) and six developed MDS-ALs. Six leukemic transformations occurred in the before-2005 group and none after 2005 (respective frequencies $3.8 \times 10^{-3}$ vs. $0 ; P<0.01$ ), while four HSCTs were done before 2005 and 12 since 2005 (respective HSCT rates increased $2.5 \times 10^{-3}$ vs. $9 \times 10^{-3} ; P<0.01$ ). Our results support early HSCT for patients with ELANE mutations who received high GCSF doses, as it might lower the risk of leukemic transformation.
\end{abstract}

\section{Introduction}

Congenital neutropenia $(\mathrm{CN})$ is a group of rare and inherited hematological disorders characterized by

Jean Donadieu

jean.donadieu@trs.aphp.fr

1 Registre des Neutropénies Chroniques, Centre de Référence des Neutropénies Chroniques, Sorbonne Université, Inserm, Centre de Recherche Saint-Antoine, Hôpital Trousseau, APHP, Paris F-75012, France

2 IRCCS Giannina Gaslini and Università degli Studi di Genova, Genoa, Italy

3 CHU de Nantes, Nantes, France

4 University of Strasbourg, Strasbourg, France

5 CHU La Timone, Marseille, France

6 Department of Pediatric Hematology/Oncology, University Hospital of Rennes, Rennes, France

7 Institut d'Hémato-Oncologie Pédiatrie IHOPE, Lyon, France

8 Necker Children's Hospital, Assistance Publique-Hôpitaux de Paris, Paris, France chronic profound neutropenia caused by impaired differentiation of neutrophilic granulocytes. Often diagnosed early in life, $\mathrm{CN}$ is defined as recurrent, life-threatening bacterial infections (e.g., skin infections, otitis, gingivitis,

9 CHU Clermont-Ferrand, Centre Régional de Cancérologie et Thérapie Cellulaire Pédiatrique, Clermont-Ferrand, France

10 Unité d'Immunologie Hématologie Pédiatrique, Necker Children's Hospital, Paris, France

11 Départment de Pédiatrie, Hémato-Oncologie, CHU de Bordeaux, Bordeaux, France

12 Départment de Pédiatrie, Hémato-Oncologie, CHU de Rouen, Rouen, France

13 Department d'Hématologie, Service de Transplantation Médullaire, Hôpital Saint-Louis, Paris, France

14 Département de Génétique, APHP, Hôpital Pitié-Salpêtrière, Sorbonne Université, Paris, France

15 Pediatric Hematology Department, Robert-Debré Hospital, Paris, France 
abscesses and sepsis) [1]. The elastase, neutrophilexpressed (ELANE) gene encodes neutrophil elastase, and is responsible for ELANE neutropenia, which is probably the most frequent and one of the most severe $\mathrm{CN}$ forms, based on its infection rate [2]. Two distinct ELANE-related $\mathrm{CN}$ phenotypes have been described: cyclic neutropenia $(\mathrm{CyN})$, in which neutrophil counts classically oscillate with 21-day periodicity, and severe congenital neutropenia ( $\mathrm{SCN}$ ), with persistently low blood neutrophil counts [3, 4]. The standard-of-care for ELANEneutropenia patients consists of granulocytecolony-stimulating factor (GCSF) administration, which increases the numbers of circulating neutrophils, thereby lowering infection-related mortality. Hematopoietic stemcell transplantation (HSCT) is indicated for the rare patients with GCSF-refractory disease (i.e., no absolute neutrophil count (ANC) increase at $\geq 50 \mu \mathrm{g} / \mathrm{kg} / \mathrm{day}$ ) or those with myelodysplastic syndrome (MDS) [5-7].

Patients with ELANE-related CN are at risk of developing MDS-acute leukemia (MDS-AL). In 2005, our team showed [8] that the $\mathrm{CN}$ leukemia risk depends on two major features, the genetic defect and GCSF use, with the latter controlling the patients' infection rates. The relationship between high-dose GCSF exposure and leukemic transformation was confirmed in 2006 by the International Severe Chronic Neutropenia Registry (ISCNR), in which the USA, Canada, UK, the Netherlands, and Germany participate [9]. Based on those results, guidelines were issued in France recommending HSCT for patients receiving high-dose GCSF, while the French Severe Chronic Neutropenia Registry has continued to collect data prospectively. This practice represents an extension of the validated indication of HSCT for CN.

This study was undertaken to analyze the effect of this policy on patients with the ELANE-gene mutation, as they are currently one of the most heavily GCSF-treated. In addition, even though HSCT has been proposed for $\mathrm{CN}$ since 1980 [10], it has been reported in only 11 patients with known ELANE status thus far [11-18]. Therefore, a comprehensive review of these cases appeared useful concerning HSCT indication and its impact.

\section{Patients and methods}

\section{Registry organization and data monitoring}

The patients included in this study were all entered in the French Severe Chronic Neutropenia Registry (FSCNR). First created in 1993, with prospective enrollment of all CN types [1], the FSCNR was certified as a national registry by health authorities in 2008, and its case completeness was ascertained by verification through multiple sources. The database was approved by the French computer watchdog commission (CNIL certificate no. 97.075). Thirty-five French pediatric hematology-oncology units and $\sim 50$ adult hematology units participate in the FSCNR. A clinical research associate assured accurate data monitoring, based on annual on-site reviews of medical charts. The following parameters are currently recorded: sex, age at diagnosis, severe bacterial or fungal infections (septicemia, cellulitis, pneumonia, osteitis and liver abscess), complete blood cell counts, differential bone-marrow cell counts (smear method) and all information on HSCTs. Each patient had to provide written informed consent to be entered in the FSCNR. Several reports on the FSCNR are available elsewhere [2, 8, 19-24].

\section{Study design}

This before-after study compared patients divided into two groups according to follow-up period: "before 2005", for those born before 2005 and followed until 31/12/2004, and "after 2005", for those born after 2005 or before 2005 but still being followed after 2005 until the last update (31/05/ 2019). The main reason to separate these two periods was the HSCT-indication modification at the end of 2004. Since 2005, patients in France receiving a GCSF dose above the $15-\mu \mathrm{g} / \mathrm{kg} /$ day threshold become eligible for HSCT, in addition to patients with validated indications, such as MDS-AL or GCSF refractoriness (typically $\geq 50 \mu \mathrm{g} / \mathrm{kg} / \mathrm{day}$ ).

\section{ELANE-neutropenia genetics}

We classified variants according to American College of Medical Genetics and Genomics guidelines [25]. Only pathogenic (class 5) and probably pathogenic ELANE-gene variants (class 4) were included in this study. The patients or their parents gave written informed consent for genetic testing. Standard procedures were used to extract DNA from blood. The ELANE-gene coding sequence and exon-intron boundaries were determined either by Sanger sequencing, as described previously [2, 3], or by targeted sequencing of a gene panel including ELANE. Mutations were numbered as recommended by the Human Genome Variation Society (http://www.hgvs.org/), using the reference sequence NM_001972.2.

\section{Literature review}

To identify all publications related to ELANE-associated neutropenia and summarize reported cases, we first screened PubMed using the keywords "ELANE" "ELA2" "Severe congenital neutropenia" "Congenital neutropenia". Then the reference list of each article was checked to identify additional references and avoid duplicates. 


\section{Hematological events}

$\mathrm{AL}$, either acute myeloid leukemia (AML) or acute lymphoblastic leukemia (ALL), was defined using WHO criteria (i.e., at least $20 \%$ blast cells in bone-marrow smears). Because these patients almost always had dystrophic cytological abnormalities, MDS was diagnosed when cytological abnormalities were associated with central anemia or central thrombocytopenia requiring blood transfusion, and when clonal cytogenetic abnormalities were present. Singlestrand conformational polymorphism, as previously described [26], was used to screen for GCSF-receptor mutations, which were confirmed by sequencing. More recently, targeted next-generation sequencing was used.

\section{GCSF exposure}

To calculate GCSF exposure, we assumed dose equivalence between filgrastim and lenograstim. Because the unit dose and frequency of GCSF injections are individually tailored, each patient's overall treatment was estimated using several parameters calculated for each GCSF therapeutic period during which the dose and frequency of injections were stable: dose delivered per injection $\left(d d_{i}, \mu \mathrm{g} / \mathrm{kg}\right)$; number of injections $\left(n_{i}\right)$; cumulative dose, defined as the total dose received during the relevant period $(\mathrm{mg} / \mathrm{kg})=d d_{i} \times n_{i}$; and duration of the relevant period in days. The total cumulative dose $(\mu \mathrm{g})$ was calculated as the sum of cumulative doses for each therapeutic period, from day 1 of GCSF to the last day of follow-up. The cumulative duration of GCSF use (in years) was calculated as the sum of all therapeutic periods. For each patient, total GCSF-therapy follow-up (in years) since onset was calculated from the first injection to the last day. The time-averaged dose $(\mu \mathrm{g} / \mathrm{kg} /$ day $)$ was calculated by dividing the cumulative dose by the cumulative duration of treatment.

\section{HSCT and engraftment}

The conditioning regimen, donor, type of graft and number of cells injected were recorded. Chimerism analysis was performed by means of cytogenetic or polymerase chain reaction (PCR) amplification of microsatellites on peripheral mononuclear blood cells.

\section{Statistical methods}

Demographic, clinical, biological and therapeutic data were recorded using an Access database (Microsoft). Stata ${ }^{\circledR}$ version 13 software was used to compute all statistical analyses. The frequencies were calculated by dividing the numbers of events by the person-years observed for before and after periods. This before-after study compared medical intervention (HSCT) and medical complication (MDS-AL) frequencies between before- and after-2005 groups, using the Mantel-Haensel test [27, 28]. Categorical data were compared with Fisher's exact test and quantitative data with the Mann-Whitney test. All tests were two-tailed, with $P<$ 0.05 defining significance. For survival analyses, the endpoints were death, bone-marrow transplantation and MDS-AL onset. The Kaplan-Meier method was used to estimate the probability of survival. Survival was compared between before and after groups with the log-rank test [29].

\section{Results}

\section{Cohort description}

As of 31 March 2019, among the $1032 \mathrm{CN}$ patients enrolled in the FSCNR, 144 carry a heterozygous, pathogenic ELANE mutation. Ninety-five $(66 \%)$ patients were diagnosed with SCN and 49 (34\%) with CyN. The cohort comprised 70 males and 74 females. The median age of the entire cohort at diagnosis was 0.24 (range 0-42.9) years. Respective median ages at $\mathrm{SCN}$ or $\mathrm{CyN}$ diagnosis were 0.19 or 0.8 years $(P<0.002)$. The cumulative observation times were 1588 person-years for the 89 patients in the before-2005 group and 1327 person-years for those in the after- 2005 group ( 55 born after 2005 and 84 born before 2005 but followed since 2005). Five patients born before 2005 were not followed after 2005 because they developed MDS-AL, died or underwent HSCT. GCSF treatments were individually tailored based on the recurrence of severe infections and GCSF tolerance. For the entire cohort, 112 patients received GCSF, at a mean dose of $9.8 \mu \mathrm{g} / \mathrm{kg} / \mathrm{day}$, and 32 patients were not given GCSF. The mean (range) total doses $(\mu \mathrm{g} / \mathrm{kg})$ were $9837(0-115431)$ for the entire cohort, $12564(0-115431)$ for SCN patients and 3837 (0-25 038) for those with CyN. For the 112 treated patients, the time-averaged dose $(\mu \mathrm{g} / \mathrm{kg} / \mathrm{day})$ was $0.1-4.9$ for three (2.7\%) patients, 5-9.9 for 47 (42\%), 10-14.9 for 26 (23.2\%), $15-29.9$ for $14(12.5 \%)$ or $30-49.9$ for nine $(8.0 \%)$ and $>50 \mu \mathrm{g} / \mathrm{kg} /$ day for $13(11.6 \%)$. Maximum doses $(\mu \mathrm{g} / \mathrm{kg} /$ day) were $<5$ for $32(22.2 \%)$ patients, $5-10$ for 38 (26.4\%), $10-20$ for 32 (22.2\%), 20-30 for 26 (18\%) or $>30$ for 16 $(11.1 \%)$. Five patients' neutropenias were considered GCSF-refractory (ANC $<0.2 \mathrm{G} / \mathrm{L}$ after receiving $\geq 50 \mu \mathrm{g} / \mathrm{kg} /$ day).

Six leukemic transformations occurred, including one ALL. None of the patients followed after 2005 developed MDS-AL. For the entire cohort, the cumulative MDS-AL frequencies (SD) were $4.4 \%$ (1.9) at 10 years and $5.5 \%$ (2.2) at 20 years. Two patients initially diagnosed with MDS developed AL. No CyN patients developed MDS-AL. Diabetes insipidus with pituitary nodules was the 

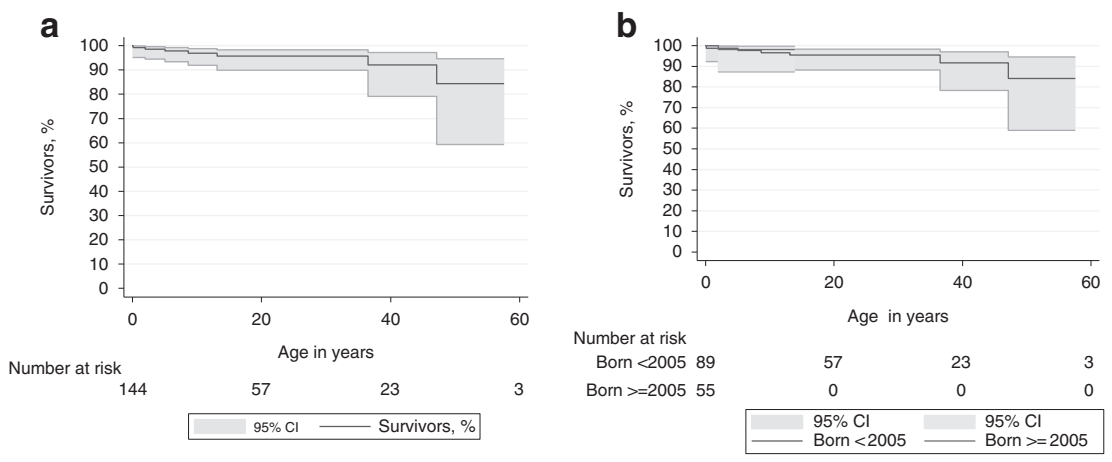

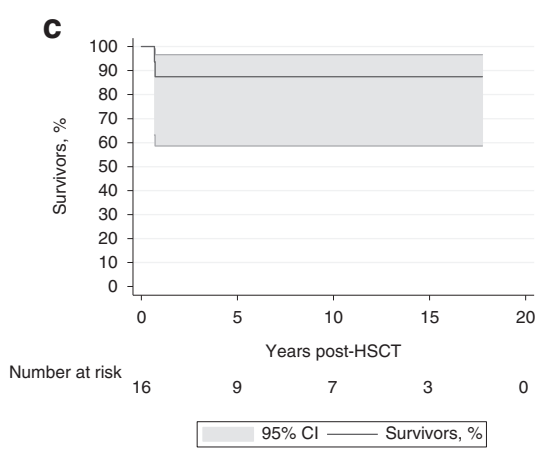

Fig. 1 Kaplan-Meier estimated probabilities of survival with $95 \%$ confidence intervals (CI). (a) the entire French Severe Chronic Neutropenia Registry cohort of 144 patients, (b) the same cohort according to their dates of birth (before 2005 or after 2005) and (c) the 16 French ELANE-neutropenia cohort patients after hematopoietic stem-cell transplantation (HSCT). first manifestation for two patients. Among the six MDS-AL patients, three had monosomy-7, and one a complex caryotype and 2 have no identified cytogenetic clone. A CSF3R mutation was detected at the time of malignant transformation in 2 patients [30]. The mean GCSF dose before MDS-AL for the six patients was $18.9 \mu \mathrm{g} / \mathrm{kg} /$ day. Median age at AL onset was 10.75 (range 4.2-12.5) years. The two patients who did not undergo HSCT died of leukemia, while, among the four transplanted patients (two for AML, one for ALL and one for MDS); patient \#3 died of HSCT-related complications, despite complete engraftment.

Seven of the 144 cohort patients died. The 20- and 40year overall survival rates $(95 \%$ CI $)$ were $95.7 \%$ (90-98.2\%) and $92.4 \% \quad(79.1-97.2 \%)$, respectively (Fig. 1b). Five nontransplanted patients died. Before 2005, two deaths were attributed to AL complications without HSCT; another died of sepsis. After 2005, one adult died of sepsis during a chemotherapy regimen for colon cancer and another died of septic shock and renal failure.

\section{Description of the 16 patients who underwent HSCT}

Six males and ten females underwent HSCT (Table 1). Median age at transplantation was 4.3 (range 0.6-20.4) years. All 16 underwent preparatory myeloablation: nine were given a combination of busulfan, cyclophosphamide and anti-thymocyte globulin (ATG); six were given fludarabine and busulfan, combined with ATG alone for four, and melphalan and ATG for one, and thiotepa and ATG for one. One patient underwent TAM12 (total body irradiation, cytarabine and melphalan). A Gly214Arg mutation was detected in $6 / 16(37.5 \%)$ HSCT recipients. HSCTs were done in 4/89 before-2005 patients and 12/139 after-2005 patients (Table 2).

All 16 patients achieved complete hematological recovery with full engraftment, and have a normal neutrophil count after HSCT. The median time required for ANCs to reach $0.5 \times 10^{9} / \mathrm{L}$ was 21 (range $14-77$ ) days; the longest engraftment time refers to an unrelated cord blood (UCB) HSCT in patient \#3. After 9-42 (median 27) days, platelet counts reached $50 \times 10^{9} / \mathrm{L}$, without further transfusion. Despite successful engraftment, patient \#13 developed autoimmunity (antinuclear factor (ANF) titer $>1 / 640$ ), lost his graft at 18 months, and was not amenable to immunosuppression. She underwent retransplantation complicated by severe varicella zoster but nonetheless achieved good and stable engraftment and is in good health, without sequelae, 4 years later.

All surviving patients are disease-free and no longer require erythrocyte and platelet transfusions, with normalized ANCs. Complete chimerism was found in all patients.

Median follow-up post-transplantation was 5.7 years and 5-year survival rate was $87.5 \%$ [95\% confidence intervals (CI) 58.7-96.7\%] (Fig. 1c). Two patients died after HSCT: patient \#3 with ALL for which HSCT was indicated, died of sepsis before 2005 and patient \#11 with GCSF-refractory ELANE neutropenia, for which HSCT was indicated, died after 2005 of refractory grade-IV GVHD with transplantassociated thrombotic microangiopathy. In addition, six patients had grade-II GVHDs, including one with chronic skin GVHD and persistent livedo.

\section{Comparison of HSCT rates and leukemia rates before vs. after 2005}

Comparing before 2005 to after 2005, respectively, median ages were 0.44 years and 0.15 years (nonsignificant difference) at diagnosis, and 6.9 (range, 1.6-12.4) and 0.15 (range, 0.6-9.3) years at transplantation $(P=0.06)$. Median follow-up durations for the before- and after-2005 cohorts were 14.2 (range 0.1-53.4) and 10.4 (range 0.2-14.5) years, respectively (nonsignificant).

Before 2005, HSCT indications were AL for three patients and GCSF refractoriness for one, and GCSF refractoriness for six after-2005 patients: five taking high 


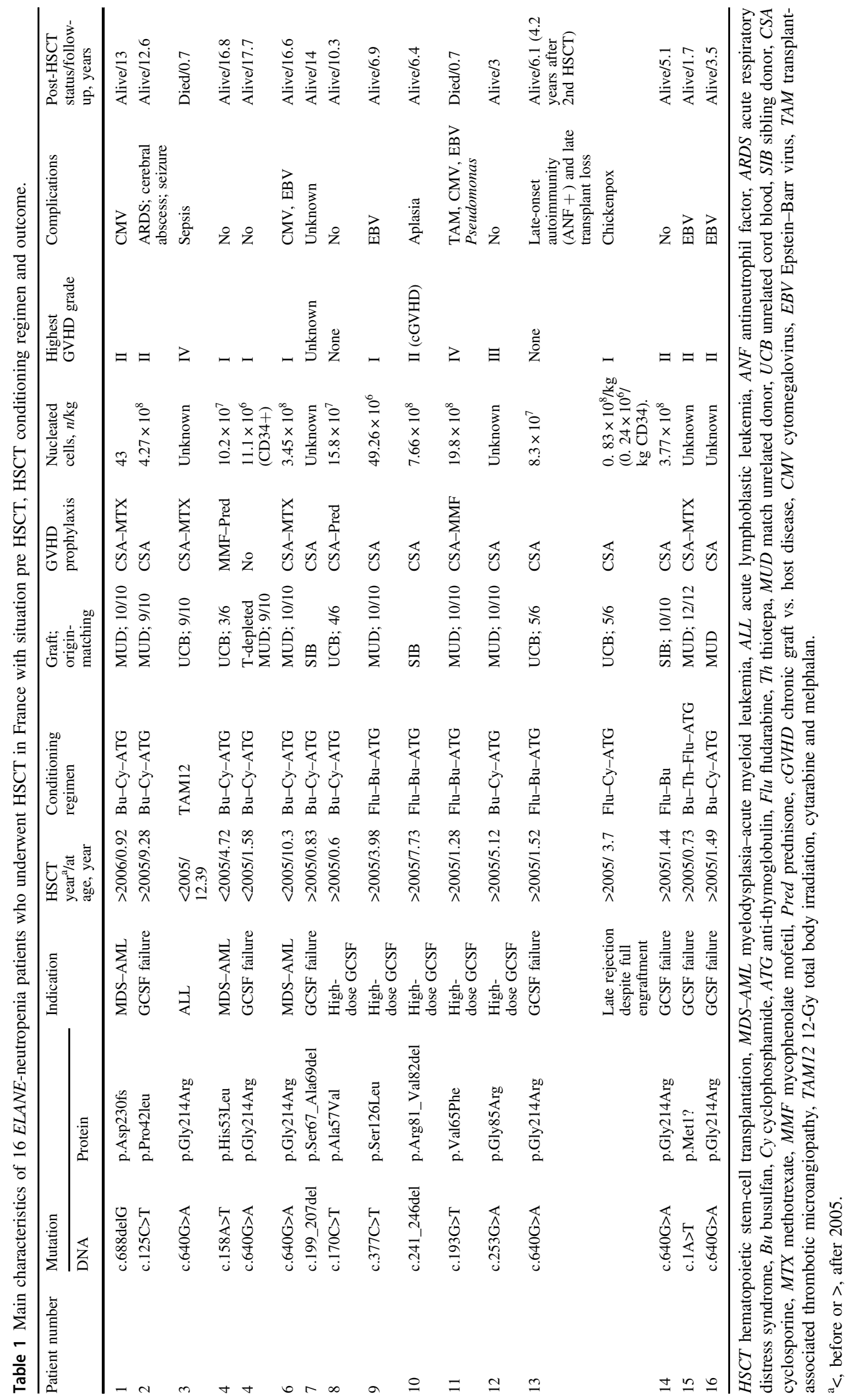


Table 2 Results by period (Before and after 2005): this before-after study compared medical intervention (HSCT) and medical complication (MDS-AL) frequencies i.e., the number of events per person-years observed, between before- and after-2005 groups, using the Mantel-Haensel test.

\begin{tabular}{lll}
\hline Parameter & Before 2005 & After 2005 to 2019 \\
\hline Patients at risk, $n$ & 89 & $139^{\mathrm{a}}$ \\
Cumulative follow-up, person-years & 1588 & 1327 \\
HSCT & & \\
$\quad 4$ & $2.5 \times 10^{-3}$ & 12 \\
$\quad$ & & $9 \times 10^{-3}$ \\
$\quad$ Ratio, person-years & 6 & $<0.01$ \\
$\quad$ MDS-AL & $3.8 \times 10^{-3}$ & 0 \\
$\quad 4[2$ AML, 1 sepsis, 1 HSCT-related] & $3[1$ colon cancer, 1 sepsis, 1 HSCT-related] \\
Incidence ratio, person-years & 6.8 & 36
\end{tabular}

HSCT hematopoietic stem-cell transplantation, $M D S-A L$ myelodysplasia-acute leukemia, $A M L$ acute myeloblastic leukemia.

a55 patients born after 2005; 84 patients born before 2005 and followed until 2019.

GCSF doses $(15-30 \mu \mathrm{g} / \mathrm{kg} / \mathrm{day})$ and one with MDS $(P<$ $0.03)$. Neither the mean before- and after-2005 GCSF doses $(8.5$ vs. $11.6 \mu \mathrm{g} / \mathrm{kg} /$ day, respectively; $P<0.09)$ nor the maximum doses (respectively, 14.4 vs. $13.6 \mu \mathrm{g} / \mathrm{kg} /$ day; $P<$ $0.15)$ received by these patients changed significantly with time. Finally, the major change between before and after 2005 was not the dose prescribed initially to treat the patients needing GCSF but the medical decision with regards to the indication of HSCT.

\section{Discussion}

The main finding issued from this population-based study on 144 patients with ELANE mutations was that slightly extending the HSCT indication to patients currently taking high-dose GCSF may lessen the risk of leukemia. In our FSCNR experience, leukemia has not been diagnosed in ELANE-neutropenia patients since 2005, when we extended the HSCT indication to patients receiving $>15 \mu \mathrm{g} / \mathrm{kg} /$ day of GCSF. Analysis of administered GCSF doses showed no differences between before-2005 and after-2005 mean doses, maximum doses or total doses for transplantation candidates. However, after 2005, the number of HSCTs increased significantly and patients were transplanted at a significantly younger mean age.

ELANE neutropenia was identified first in CyN [31], and then shortly thereafter in chronic neutropenia [32]. Infections and hematological malignancies are the main life-threatening complications of ELANE neutropenia. Long-term use of GCSF to treat CN, which started in 1988, markedly limits the infectious risk, dramatically improving these patients' quality of life. However, it has also raised questions regarding the balance between the natural risk of severe infections and that of iatrogenic leukemic transformation $[8,9]$.

Patients carrying an ELANE mutation can develop MDS, AML or, more rarely, ALL. The leukemic transformation rate reported in the literature for ELANE-neutropenia patients is $15-25 \%$ by 20 years of age [2, 3, 33]. That risk is considered to be stable over time, affecting $25 \%$ of the 52 patients in a series from the NIH and US SCN registry [33]. During the last 20 years, two large case series were reported; one from the ISCNR on 307 patients [4] and the other from the Hannover genetic laboratory on 213 patients [3]. Those reports mentioned that 40 HSCTs had been performed but no information was provided about the indications or outcomes. Among the largest series of HSCTs (136 including $39 E L A N E$-neutropenia patients) indicated for SCN [6], which includes our patients, the HSCT indication was not analyzed, and that series was not population-based. An exhaustive literature review of HSCT for CN identified only 11 cases with a known ELANE mutation (Table 3) and sufficient details about transplantation indications and outcomes) [11-18]. All the reported HSCT indications were 'classical' in the literature reviewed: three for MDS-AL and eight for GCSF refractoriness. None of those papers can be used to study the HSCT impact on the risk of developing MDS-AL, because only the numbers of HSCTs during follow-up of all ELANE-neutropenia patients are known but not the entire population.

Because our study was an exhaustive examination of a national cohort, with the participation of all French centers, we think that it could be representative of a nonbiased sample of ELANE- neutropenia patients. Our first important observation is that the HSCT outcome for ELANE- neutropenia patients was good, with overall $95 \%$ engraftment survival and $90 \%$ long-term survival. Extending HSCT to a 


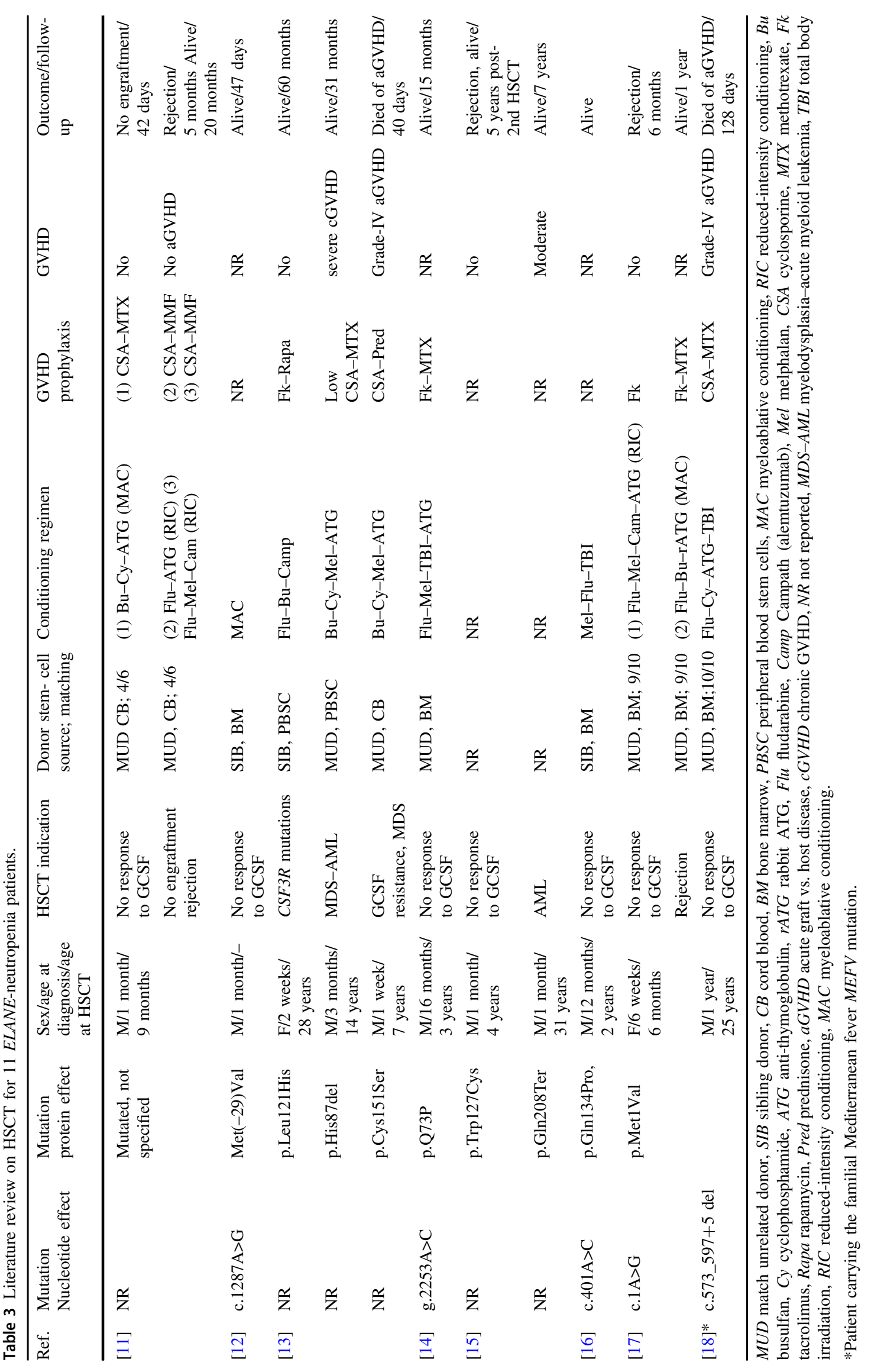


small number of patients requiring high-dose GCSF proved beneficial and may lower their risk of AL.

Many data have been collected on ELANE-neutropenia patients regarding clonal evolution. The first clonal event was shown to occur in the colony-stimulating factor-3 (CSF3R) clone [34], but the ultimate clonal evolution involved a broader panel of mutations at the time of leukemic transformation [35, 36]. Although such information is crucial to following patients with ELANE-germline mutations, the initial step of leukemogenesis in ELANEneutropenia patients involved CSF3R mutations, which appear to be induced by GCSF therapy, and is sufficient to trigger MDS-AL. Taking into consideration the GCSF 'load', key information on early patient management may potentially help make the clinical decision about the HSCT indication.

Our results are limited by the small sample size. Further studies on larger populations could help improve our understanding the GCSF role and what dose can reasonably be considered low risk for leukemic transformation. However, HSCT remains a viable therapeutic option for patients who are difficult to manage and at high risk of severe infections.

Acknowledgements The French Severe Chronic Neutropenia Registry is supported by grants from Amgen SAS, Chugai SA, GIS Maladies Rares and Inserm. FSCNR has been supported by constant and unlimited funding from the Association Sportive de SaintQuentin-Fallavier since 2004 with the unlimited commitment of $\mathrm{Mr}$ Gonnot. The authors thank the patients and their families for their participation in this study, the association IRIS and Mrs Grosjean for her support. This study was conducted by the Centre de Reference des Neutropénies Chroniques (CRNC, www.neutropenie.fr) with Société d'Hémato-Immunologie Pédiatrique support. The authors thank Ms Janet Jacobson for editorial assistance.

Author contributions The original design of the study was conceived by JD and GAR. JD is the coordinator of the FSCNR and BB is responsible for data management. $\mathrm{CB}-\mathrm{C}$ ran the genetic analyses. All the authors contributed to writing manuscript and its revision.

\section{Compliance with ethical standards}

Conflict of interest The authors declare that they have no conflict of interest.

Publisher's note Springer Nature remains neutral with regard to jurisdictional claims in published maps and institutional affiliations.

\section{References}

1. Donadieu J, Beaupain B, Fenneteau O, Bellanne-Chantelot C. Congenital neutropenia in the era of genomics: classification, diagnosis, and natural history. Br J Haematol. 2017;179:557-74.

2. Bellanne-Chantelot $\mathrm{C}$, Clauin $\mathrm{S}$, Leblanc $\mathrm{T}$, Cassinat $\mathrm{B}$, Rodrigues-Lima F, Beaufils S, et al. Mutations in the ELA2 gene correlate with more severe expression of neutropenia: a study of
81 patients from the French Neutropenia Register. Blood. 2004;103:4119-25.

3. Germeshausen M, Deerberg S, Peter Y, Reimer C, Kratz CP, Ballmaier M. The spectrum of ELANE mutations and their implications in severe congenital and cyclic neutropenia. Hum Mutat. 2013;34:905-14.

4. Makaryan V, Zeidler C, Bolyard AA, Skokowa J, Rodger E, Kelley ML, et al. The diversity of mutations and clinical outcomes for ELANE-associated neutropenia. Curr Opin Hematol. 2015;22:3-11.

5. Ferry C, Ouachee M, Leblanc T, Michel G, Notz-Carrere A, Tabrizi R, et al. Hematopoietic stem cell transplantation in severe congenital neutropenia: experience of the French SCN register. Bone Marrow Transplant. 2005;35:45-50.

6. Fioredda F, Iacobelli S, van BA, Gaspar B, Ancliff P, Donadieu J, et al. Stem cell transplantation in severe congenital neutropenia: an analysis from the European Society for Blood and Marrow Transplantation. Blood. 2015;126:1885-92.

7. Zeidler C, Welte K, Barak Y, Barriga F, Bolyard AA, Boxer L, et al. Stem cell transplantation in patients with severe congenital neutropenia without evidence of leukemic transformation. Blood. 2000;95:1195-8.

8. Donadieu J, Leblanc T, Bader MB, Barkaoui M, Fenneteau O, Bertrand $\mathrm{Y}$, et al. Analysis of risk factors for myelodysplasias, leukemias and death from infection among patients with congenital neutropenia. Experience of the French Severe Chronic Neutropenia Study Group. Haematologica. 2005;90:45-53.

9. Rosenberg PS, Alter BP, Bolyard AA, Bonilla MA, Boxer LA, Cham $B$, et al. The incidence of leukemia and mortality from sepsis in patients with severe congenital neutropenia receiving long-term G-CSF therapy. Blood. 2006;107:4628-35.

10. Rappeport JM, Parkman R, Newburger P, Camitta BM, Chusid MJ. Correction of infantile agranulocytosis (Kostmann's syndrome) by allogeneic bone marrow transplantation. Am J Med. 1980;68:605-9.

11. Markel MK, Haut PR, Renbarger JA, Robertson KA, Goebel WS. Unrelated cord blood transplantation for severe congenital neutropenia: report of two cases with very different transplant courses. Pediatr Transplant. 2008;12:896-901.

12. Setty BA, Yeager ND, Bajwa RP. Heterozygous M1V variant of ELA-2 gene mutation associated with G-CSF refractory severe congenital neutropenia. Pediatr Blood Cancer. 2011;57:514-5.

13. Carlsson G, Winiarski J, Ljungman P, Ringden O, Mattsson J, Nordenskjold M, et al. Hematopoietic stem cell transplantation in severe congenital neutropenia. Pediatr Blood Cancer. 2011; $56: 444-51$.

14. Kawaguch K, Matsubara K, Uchida Y, Saito A, Miyata K, Hasegawa D, et al. Successful treatment with allogenic hematopoietic stem cell transplantation of a severe congenital neutropenia patient harboring a novel ELANE mutation. Rinsho Ketsueki. 2014;55:2294-9.

15. Lebel A, Yacobovich J, Krasnov T, Koren A, Levin C, Kaplinsky $\mathrm{C}$, et al. Genetic analysis and clinical picture of severe congenital neutropenia in Israel. Pediatr Blood Cancer. 2015;62:103-8.

16. Nino N, Kozaki A, Hasegawa D, Ueda G, Takahashi H, Miyata K, et al. Successful non-myeloablative allogenic bone marrow transplantation in a child with severe congenital neutropenia complicated by chronic pulmonary infection. Rinsho Ketsueki. 2016;57:742-7.

17. Hashem H, bu-Arja R, Auletta JJ, Rangarajan HG, Varga E, Rose $\mathrm{MJ}$, et al. Successful second hematopoietic cell transplantation in severe congenital neutropenia. Pediatr. Transplant. 2018;22:e13078.

18. Okolo ON, Katsanis E, Yun S, Reveles CY, Anwer F. Allogeneic transplant in ELANE and MEFV mutation positive severe cyclic neutropenia: review of prognostic factors for secondary severe events. Case Rep Hematol. 2017;2017:5375793. 
19. Donadieu J, Michel G, Merlin E, Bordigoni P, Monteux B, Beaupain B, et al. Hematopoietic stem cell transplantation for Shwachman-Diamond syndrome: experience of the French neutropenia registry. Bone Marrow Transplant. 2005;36:787-92.

20. Beaussant CS, Fenneteau O, Plouvier E, Rohrlich PS, Daltroff G, Plantier I, et al. Description and outcome of a cohort of 8 patients with WHIM syndrome from the French Severe Chronic Neutropenia Registry. Orphanet J Rare Dis. 2012;7:71.

21. Desplantes C, Fremond M, Beaupain B, Harousseau J, Buzyn A, Pellier I, et al. Clinical spectrum and long-term follow-up of 14 cases with G6PC3 mutations from the French severe congenital neutropenia registry. Orphanet J Rare Dis. 2014;9:183.

22. Donadieu J, Fenneteau O, Beaupain B, Beaufils S, Bellanger F, Mahlaoui N, et al. Classification of and risk factors for hematologic complications in a French national cohort of 102 patients with Shwachman-Diamond syndrome. Haematologica. 2012;97: 1312-9.

23. Donadieu J, Lamant M, Fieschi C, de Fontbrune FS, Caye A, Ouachee M, et al. Natural history of GATA2 deficiency in a survey of 79 French and Belgian patients. Haematologica. 2018;103:1278-87.

24. Rigaud C, Lebre AS, Touraine R, Beaupain B, Ottolenghi C, Chabli A, et al. Natural history of Barth syndrome: a national cohort study of 22 patients. Orphanet J Rare Dis. 2013;8:70.

25. Richards S, Aziz N, Bale S, Bick D, Das S, Gastier-Foster J, et al. Standards and guidelines for the interpretation of sequence variants: a joint consensus recommendation of the American College of Medical Genetics and Genomics and the Association for Molecular Pathology. Genet Med. 2015;17:405-24.

26. Dong F, Dale DC, Bonilla MA, Freedman M, Fasth A, Neijens $\mathrm{HJ}$, et al. Mutations in the granulocyte colony-stimulating factor receptor gene in patients with severe congenital neutropenia. Leukemia. 1997;11:120-5.
27. Martin DO, Austin H. An exact method for meta-analysis of casecontrol and follow-up studies. Epidemiology. 2000;11:255-60.

28. Miettinen O. Estimability and estimation in case-referent studies. Am J Epidemiol. 1976;103:226-35.

29. Collet D. Modelling survival data in medical research. London: Chapman and Hall; 1994.

30. Cassinat B, Bellanne-Chantelot C, Notz-Carrere A, Menot ML, Vaury C, Micheau M, et al. Screening for G-CSF receptor mutations in patients with secondary myeloid or lymphoid transformation of severe congenital neutropenia. A report from the French neutropenia register. Leukemia. 2004;18:1553-5.

31. Horwitz M, Benson KF, Person RE, Aprikyan AG, Dale DC. Mutations in ELA2, encoding neutrophil elastase, define a 21-day biological clock in cyclic haematopoiesis. Nat Genet. 1999; 23:433-6.

32. Dale DC, Person RE, Bolyard AA, Aprikyan AG, Bos C, Bonilla $\mathrm{MA}$, et al. Mutations in the gene encoding neutrophil elastase in congenital and cyclic neutropenia. Blood. 2000;96:2317-22.

33. Rosenberg PS, Alter BP, Link DC, Stein S, Rodger E, Bolyard AA, et al. Neutrophil elastase mutations and risk of leukaemia in severe congenital neutropenia. Br J Haematol. 2008;140:210-3.

34. Germeshausen M, Ballmaier M, Welte K. Incidence of CSF3R mutations in severe congenital neutropenia and relevance for leukemogenesis: Results of a long-term survey. Blood. 2007; 109:93-9.

35. Beekman R, Valkhof MG, Sanders MA, van Strien PM, Haanstra JR, Broeders L, et al. Sequential gain of mutations in severe congenital neutropenia progressing to acute myeloid leukemia. Blood. 2012;119:5071-7.

36. Skokowa J, Steinemann D, Katsman-Kuipers JE, Zeidler C, Klimenkova O, Klimiankou M, et al. Cooperativity of RUNX1 and CSF3R mutations in severe congenital neutropenia: a unique pathway in myeloid leukemogenesis. Blood. 2014;123:2229-37. 